\title{
LC Headings from July and August 2019 Lists
}

Compiled by Ann Heinrichs

The new headings listed here reflect the most recent information available at the time of publication. Items in this list were selected from 2019 list numbers 07 (July 12) and 08 (August 16).

\section{SUBJECT HEADINGS}

150 Anglican authors [May Subd Geog] [sp2019000797]

360 SA subdivision Anglican authors under names of literatures, e.g., English literature-Anglican authors

550 BT Authors

150 Antidepressants--Religious aspects [sp2019100010]

150 Antidepressants--Religious aspects--Catholic Church [sp2019100009]

150 Bible stories, Karo-Batak [May Subd Geog] [sp2019000831]

450 UF Karo-Batak Bible stories

150 Black Virgins CHANGE HEADING

150 Black Madonnas [May Subd Geog] [sp 85014640]

053 BT670.B55

450 UF Black Virgins [Former heading]

450 UF Madonnas, Black

450 UF Virgins, Black

500 BT Mary, Blessed Virgin, Saint--Art

500 BT Mary, Blessed Virgin, Saint--Devotion to

150 Hymns, Bobangi [May Subd Geog] [sp2019004545]

450 UF Bobangi hymns

150 Catholic families [May Subd Geog] [sp2019003702]

550 BT Families

150 Clergy [May Subd Geog] [sp 85026944]

$450 \quad$ UF Diocesan clergy ADD FIELD

450 UF Ecclesiastics ADD FIELD

450 UF Secular clergy ADD FIELD

150 Lai Haraoba (Festival) [May Subd Geog] [sp2019000716]

450 UF Laiharaoba (Festival) 
450 UF Umālai Harāuba (Festival)

450 UF Umaṃlāi Harāuba (Festival)

450 UF Umang-Lai Haraoba (Festival)

450 UF Umangli Haraoba (Festival)

550 BT Festivals--India

550 BT Meitheis (Indic people)--Rites and ceremonies

150 Melancholy--Religious aspects [sp2019100012]

150 Melancholy--Religious aspects--Christianity [sp2019100011]

150 Mizrahim in motion pictures [Not Subd Geog] [sp2019000192]

053 PN1995.9.J46

550 BT Motion pictures

680 Here are entered works on the depiction of Mizrahim in motion pictures.

150 Mizrahim on television [Not Subd Geog] [sp2019100144]

550 BT Television

680 Here are entered works on the depiction of Mizrahim on television.

150 Ontology--Religious aspects [sp2019004510]

150 Ontology--Religious aspects--Islam [sp2019004511]

150 Pilgrims and pilgrimages (Islamic law) [May Subd Geog] [sp2019004532]

053 KBP184.7-KBP184.73

550 BT Islamic law

150 Psychic trauma--Religious aspects [sp2019100004]

150 Wiccan ethics [May Subd Geog] [sp2019005206]

$550 \quad$ BT Religious ethics

150 Zapopan, Our Lady of [Not Subd Geog] [sp2019003700]

$053 \quad$ BT660.Z3

450 UF Expectación de Zapopan, Nuestra Señora de la

450 UF Expectation of Zapopan, Our Lady of the

450 UF Nuestra Señora de la Expectación de Zapopan

450 UF Nuestra Señora de Zapopan

450 UF Our Lady of the Expectation of Zapopan

450 UF Our Lady of Zapopan

450 UF Virgen de Zapopan

500 BT Mary, Blessed Virgin, Saint--Apparitions and miracles--Mexico

500 BT Mary, Blessed Virgin, Saint--Devotion to--Mexico 